\title{
Early versus Traditional Postoperative Oral Feeding in Patients Undergoing Elective Colorectal Surgery: A Meta-Analysis of Randomized Clinical Trials
}

\author{
Cheng-Le Zhuang ${ }^{\mathrm{a}}$ Xing-Zhao Ye ${ }^{\mathrm{a}}$ Chang-Jing Zhang ${ }^{\mathrm{a}}$ Qian-Tong Dong ${ }^{\mathrm{a}}$ \\ Bi-Cheng Chen ${ }^{b}$ Zhen $\mathrm{Yu}^{\mathrm{a}}$ \\ ${ }^{a}$ Department of Gastrointestinal Surgery and ${ }^{b}$ Wenzhou Key Laboratory of Surgery, The First Affiliated Hospital, \\ Wenzhou Medical College, Wenzhou, China
}

\section{Key Words}

Early oral feeding - Traditional feeding $\cdot$ Colorectal surgery .

Postoperative complications · Outcomes

\begin{abstract}
Background: The safety and effectiveness of early oral feeding after colorectal surgery has not been determined. We performed a meta-analysis to evaluate surgical outcomes following early oral feeding compared with traditional oral feeding in patients undergoing elective colorectal surgery. Methods: MEDLINE, EMBASE, and the Cochrane Central Register of Controlled Trials were searched to identify randomized clinical trials comparing the outcomes following early oral feeding versus traditional oral feeding in patients undergoing elective colorectal surgery. The trials must have reported at least one of the following end points: anastomotic dehiscence, pneumonia, wound infection, nasogastric tube reinsertion, vomiting, mortality, length of hospital stay, hospital costs, and quality of life. Results: Seven trials, which included a total of 587 patients, met our inclusion criteria. Compared with traditional oral feeding, early oral feeding reduced the length of hospital stay (weighted mean difference -1.58 days; $95 \% \mathrm{Cl}-2.77$ to $-0.39 ; \mathrm{p}=0.009$ ) and the total postoperative complications (relative risk $0.70 ; 95 \% \mathrm{Cl}$ $0.50-0.98 ; p=0.04)$. There were no significant differences in
\end{abstract}

the risk of anastomotic dehiscence, pneumonia, wound infection, rate of nasogastric tube reinsertion, vomiting, or mortality. Conclusions: Early oral feeding is safe and effective in patients undergoing elective colorectal surgery.

(c) 2013 S. Karger AG, Basel

\section{Introduction}

Traditionally, the postoperative management of patients undergoing gastrointestinal (GI) surgery has been to keep them 'nil by mouth' and provide gastric decompression via a nasogastric tube (NGT) until the postoperative ileus resolves and bowel function resumes [1]. This management has been adopted over the years with the notion that restriction of oral feeding gives the GI tract more time to heal and recover, thus reducing postoperative complications [1, 2]. However, clinical trials do not support this. There is evidence from a growing number of randomized clinical trials (RCTs) showing that early feeding is safe and effective in patients undergoing elective GI surgery. Additionally, meta-analyses on this topic have concluded that early feeding is tolerable and benefi-

C.-L. Zhuang and X.-Z. Ye are co-first authors and contributed equally to this work.

\section{KARGER}

E-Mail karger@karger.com

www.karger.com/dsu
(C) 2013 S. Karger AG, Basel

0253-4886/13/0303-0225\$38.00/0
Zhen $\mathrm{Yu}, \mathrm{MD}, \mathrm{PhD}$

Department of Gastrointestinal Surgery

The First Affiliated Hospital of Wenzhou Medical College

2 Fuxue Lane, Wenzhou, Zhejiang Province 325000 (China)

E-Mail yuzhen0577@gmail.com 
cial to the patient [3-6]. The routes of feeding include oral and enteral tubes, but the latter could lead to many complications, including patient discomfort, tube malposition, aspiration pneumonia, sinusitis, epistaxis, and tube occlusion [7-9]. For example, tube occlusion is a common complication of enteral feeding tubes, with an incidence as frequent as $23-35 \%$ [10]. Those complications may influence postoperative outcomes. However, all previous meta-analyses have combined studies of oral feeding and tube feeding. Therefore, it was necessary to conduct a meta-analysis of RCTs involving oral feeding only. Moreover, new RCTs involving early oral feeding (EOF) in patients undergoing elective GI surgery have been published in recent years. Since most of these trials involved colorectal surgery, we performed a meta-analysis to evaluate the surgical outcomes following EOF compared with traditional oral feeding (TOF) in patients undergoing elective colorectal surgery. The present meta-analysis was performed in consistency with the recommendations of the Preferred Reporting Items for Systematic Reviews and Meta-Analyses (PRISMA) statement [11].

\section{Methods}

\section{Literature Search}

RCTs published between January 1966 and March 2013 were searched in PubMed, EMBASE, and the Cochrane Central Register of Controlled Trials. No language restrictions were applied. To ensure that no clinical trials were overlooked, the reference lists of identified articles, previous meta-analyses, and review articles were manually searched to identify additional studies. Article titles and abstracts were screened, and full texts were reviewed independently by 2 reviewers (C.-L.Z. and X.-Z.Y.); discrepancies were resolved by discussion between the reviewers. We used the following search strategy for PubMed: ((early OR immediate*) AND (oral OR enteral) AND (feed* OR nutrition OR diet)) AND ((colorect* OR colo* OR rect* OR sigmoid OR bowel OR intestin* OR 'Colorectal Neoplasms'[Mesh] OR 'cecal neoplasms' $[\mathrm{MeSH}]$ ) AND (resection OR surgery OR surgical OR laparoscop*) OR ('laparoscopy' [MeSH] OR 'Laparotomy'[Mesh] OR 'Colorectal Surgery'[MeSH] OR 'Colectomy'[MeSH] OR 'Colon/surgery'[MeSH] OR 'Colonic Diseases/surgery'[Mesh] OR 'Rectal Diseases/surgery'[Mesh] OR 'Rectum/surgery' [Mesh])) AND (randomized controlled trial[pt] OR randomized[tiab] OR placebo[tiab] OR clinical trials as topic[mesh:no exp] OR randomly[tiab] OR trial[ti]) NOT (animals[mh] NOT humans[mh])).

\section{Inclusion and Exclusion Criteria}

All published RCTs that compared early and traditional feeding in patients undergoing elective colorectal surgery were considered. The route of feeding was oral, not tube feeding. We defined EOF as any oral caloric intake commencing within $24 \mathrm{~h}$ postoperatively. TOF was defined as withholding oral intake until passage of flatus or bowel movement or more than $24 \mathrm{~h}$ postoperatively. Studies were required to report at least one of the outcome measures mentioned below. When more than one version of the same study was found, only the most recent version was included. Exclusion criteria included tube feeding, emergency surgery, parenteral nutrition, use of immune-enhancing feeding products, fast-track programs, including other interventions that might influence postoperative outcomes except EOF, inability to identify whether feeding was given within $24 \mathrm{~h}$, or no data available for the present meta-analysis.

\section{Data Extraction and Outcomes}

Two reviewers (C.-L.Z. and X.-Z.Y.) independently reviewed all eligible studies and extracted data; discrepancies were resolved through discussion between the reviewers and through reference to the original articles. We attempted to contact the study authors for additional information when necessary. Extracted information from each eligible study included: (1) study information including the name of the first author, year of publication, and number of patients in each group; (2) patient information including age, gender, and type of surgery, and (3) EOF protocol and outcome measures.

Primary outcome measures included: (1) length of postoperative hospital stay (defined as the number of days in the hospital after surgery until discharge); (2) total postoperative complications (defined as any complication reported within the postoperative period, excluding mortality and nausea/vomiting); (3) anastomotic dehiscence; (4) pneumonia, and (5) wound infection.

Secondary outcome measures included: (1) vomiting, (2) NGT reinsertion; (3) mortality within 30 days postoperatively; (4) hospital costs, and (5) quality of life. All outcomes assessed were clinically relevant in the context of colorectal surgery.

\section{Assessment of Methodological Quality}

Two authors (C.-L.Z. and X.-Z.Y.) independently evaluated the quality of the methodology of each study using the Jadad scoring system, which assesses descriptions of randomization, blinding, and withdrawals or dropouts [12]. The quality scale ranges from 0 to 5 , with a low-quality report receiving a score of 2 or less and a high-quality report receiving a score of 3 or more. Disagreement was resolved through consensus and discussion.

\section{Statistical Analysis}

Meta-analyses were performed using relative risk (RR) for dichotomous outcomes and weighted mean difference (WMD) for continuous outcome measures. Pooled estimates were presented with $95 \%$ CI. The presence and amount of heterogeneity were assessed with a $\mathrm{Q}$ test and the $\mathrm{I}^{2}$ index, and $\mathrm{p}<0.1$ was considered statistically significant $[13,14]$. A random effects model was used for pooling when there was evidence of heterogeneity; otherwise, a fixed effects model was used. Funnel plots were created to determine the presence of publication bias, and the asymmetry of each funnel plot was evaluated with the Egger weighted linear regression test, with $\mathrm{p}<0.1$ considered statistically significant [15]. For all other comparisons, statistical significance was defined by $\mathrm{p}<$ 0.05 , and all tests were 2 -sided. Data analysis was performed with Review Manager software version 5.1 from the Cochrane Collaboration and STATA version 12.0 (StataCorp, College Station, Tex., USA). Some outcomes were not analyzed but are presented in a descriptive way. 
Fig. 1. Selection process for studies included in the meta-analysis.

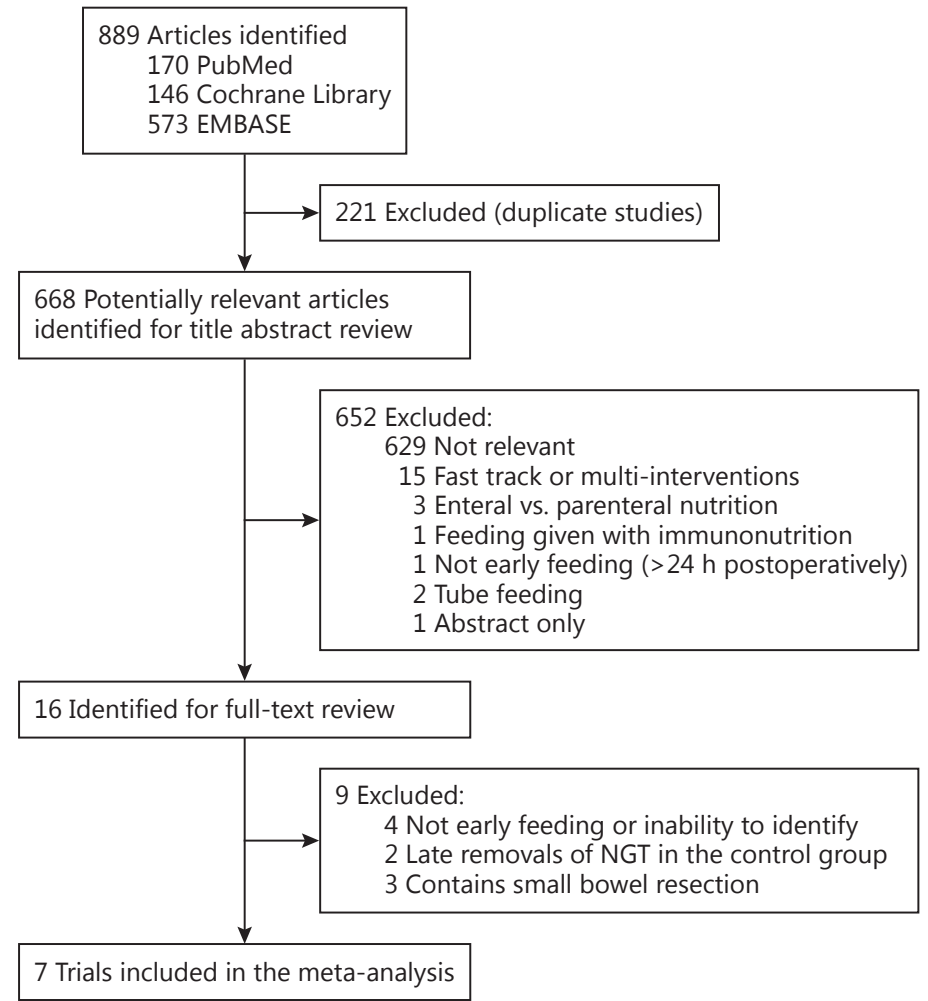

Table 1. Characteristics of randomized clinical trials of EOF after GI surgery included in the meta-analysis

\begin{tabular}{|c|c|c|c|c|c|c|c|c|c|c|c|}
\hline \multirow[t]{2}{*}{ Source } & \multicolumn{2}{|c|}{ Patients, $\mathrm{n}$} & \multicolumn{2}{|c|}{ Mean age (range), years } & \multicolumn{2}{|c|}{ Male sex, \% } & \multirow[t]{2}{*}{ Type of surgery } & \multicolumn{4}{|c|}{ Jadad scores } \\
\hline & EOF & TOF & $\mathrm{EOF}$ & TOF & EOF & TOF & & $\mathrm{R}$ & $\mathrm{B}$ & $\mathrm{W} / \mathrm{D}$ & $\mathrm{T}$ \\
\hline Dag et al. [22] & 99 & 100 & $62(35-85)$ & $61(17-89)$ & 53 & 61 & AR, LA, LH, RH, SC, SR, TC, VLA. All LT & 2 & 0 & 1 & 3 \\
\hline da Fonseca et al. [21] & 24 & 26 & $57.4(16.3)$ & $51.7(13.3)$ & 33.3 & 38.4 & LH, RH, TC, TTC. LT and LS & 2 & 0 & 1 & 3 \\
\hline $\begin{array}{l}\text { Lobato Dias Consoli } \\
\text { et al. [20] }\end{array}$ & 15 & 14 & $54.5(35-75)$ & $47.4(21-79)$ & 27 & 36 & LH, RH, SR, TC, TTC. LT and LS & 1 & 0 & 0 & 1 \\
\hline El Nakeeb et al. [19] & 60 & 60 & $52.3(21-70)$ & $56.3(25-69)$ & 65 & 42 & CC, LA, LH, RH. All LT & 2 & 0 & 1 & 3 \\
\hline Lucha et al. [18] & 26 & 25 & $51(22-74)$ & $51(22-74)$ & 65 & 65 & APR, CC, LA, LH, PC, RH, SC, TTC. All LT & 1 & 0 & 1 & 2 \\
\hline Stewart et al. [17] & 40 & 40 & $58(25-89)$ & $59(17-88)$ & 47.5 & 45 & AR, ICR, LH, RH, SC. All LT & 2 & 0 & 1 & 3 \\
\hline Hartsell et al. [16] & 29 & 29 & $66(22-82)$ & $68(40-83)$ & NR & NR & APR, CC, ICR, RCC, TPC. All LT & 1 & 0 & 0 & 1 \\
\hline
\end{tabular}

$\mathrm{NR}=$ Not reported $\mathrm{R}=$ randomization $\mathrm{B}=$ blinding; $\mathrm{W} / \mathrm{D}=$ withdrawals/dropouts $\mathrm{T}=$ total; $\mathrm{AR}=$ anterior resection; $\mathrm{APR}=$ abdominoperineal resec tion; $\mathrm{CC}=$ closure of colostomy; ICR = ileocolic resection; $\mathrm{LA}=$ low anterior resection; $\mathrm{LH}=$ left hemicolectomy; $\mathrm{PC}=$ proctectomy; $\mathrm{RCC}=$ resection with colocolostomy; $\mathrm{RH}=$ right hemicolectomy; $\mathrm{SC}=$ subtotal colectomy; $\mathrm{SR}=$ sigmoid resection; $\mathrm{TC}=$ transverse colectomy; $\mathrm{TPC}=$ total proctocolectomy; TTC = total colectomy; VLA = very low anterior resection; LT = laparotomy; LS = laparoscopy.

\section{Results}

Of 668 potentially relevant studies identified in the initial literature search, 7 studies with a total of 587 patients were included in the meta-analysis (fig. 1) [16-22].
Table 1 describes the characteristics and methodological quality (Jadad scores) of trials included in the metaanalysis. None of the included studies achieved a modified Jadad score greater than 3 (range 1-3, mean 2.3). Four studies described the method of randomization and 
Table 2. Early feeding protocol and primary outcomes of the included studies

\begin{tabular}{|c|c|c|}
\hline Source & EOF protocol & Outcomes \\
\hline Dag et al. [22] & $\begin{array}{l}\text { Fluid diet } 12 \mathrm{~h} \text { after the operation, gradually } \\
\text { increased to a solid diet as tolerated }\end{array}$ & $\begin{array}{l}\text { Length of hospital stay, complications such as wound } \\
\text { infection }\end{array}$ \\
\hline da Fonseca et al. [21] & $\begin{array}{l}\text { Received an oral liquid diet (approximately } \\
500 \mathrm{~cm}^{3} \text { ) on POD 1, and regular diet within the } \\
\text { next } 24 \mathrm{~h} \text {, as tolerated and at their discretion }\end{array}$ & $\begin{array}{l}\text { Length of hospital stay, time to first flatus and } \\
\text { defecation, complications such as anastomotic leak, } \\
\text { wound infection, pulmonary; mortality rate }\end{array}$ \\
\hline El Nakeeb et al. [19] & $\begin{array}{l}\text { Began fluids on POD } 1 \text { and advanced to a } \\
\text { regular diet within the next } 24-48 \mathrm{~h} \text { as } \\
\text { tolerated }\end{array}$ & $\begin{array}{l}\text { Length of hospital stay, time to passage of first flatus, } \\
\text { wound complication, anastomotic leakage, pulmonary } \\
\text { infection }\end{array}$ \\
\hline Stewart et al. [17] & $\begin{array}{l}\text { Free fluids from } 4 \mathrm{~h} \text { after the operation and } \\
\text { progressed to a solid diet from POD } 1 \text { at their } \\
\text { own discretion }\end{array}$ & $\begin{array}{l}\text { Tube reinsertion, vomiting, various complications } \\
\text { reported, time to first passage of flatus }\end{array}$ \\
\hline Hartsell et al. [16] & Began a full liquid diet on POD 1 & $\begin{array}{l}\text { Nausea, vomiting, complications, infection, length of } \\
\text { hospital stay }\end{array}$ \\
\hline
\end{tabular}

reported withdrawals. None of the included studies used blinding for the observers or patients. Table 2 describes the early feeding protocol and outcomes of the included studies.

\section{Primary Outcome Measures}

Length of hospital stay was assessed in all included studies. Two of the studies did not report the mean or SD for this outcome; authors were contacted for additional information but did not respond [18, 20]. Thus, the analysis for length of hospital stay was based on 5 trials. Length of hospital stay was significantly reduced for the EOF group (WMD -1.58 days; $95 \%$ CI -2.77 to -0.39 days; $\mathrm{p}=0.009$, from a random effects model), with some evidence of heterogeneity between trials $\left(\chi^{2}=18.37, \mathrm{p}=\right.$ $0.001, \mathrm{I}^{2}=78 \%$ ) (fig. 2).

Anastomotic dehiscence was assessed in 6 studies. EOF did not reduce or increase the risk of anastomotic dehiscence compared with TOF (RR 0.47; 95\% CI 0.19$1.15 ; \mathrm{p}=0.1$, from a fixed effects model), with no heterogeneity between trials $\left(\chi^{2}=3.67, \mathrm{p}=0.6, \mathrm{I}^{2}=0 \%\right)$.
Pneumonia was assessed in 6 studies. EOF did not reduce or increase the risk of pneumonia compared with TOF (RR 0.71; 95\% CI 0.31-1.59; $\mathrm{p}=0.4$, from a fixed effects model), with no heterogeneity between trials $\left(\chi^{2}=\right.$ $\left.3.53, \mathrm{p}=0.62, \mathrm{I}^{2}=0 \%\right)$.

Wound infection was assessed in 4 studies. EOF did not reduce or increase the risk of wound infection compared with TOF (RR 0.69; 95\% CI 0.34-1.37; $\mathrm{p}=0.29$, from a fixed effects model), with no heterogeneity between trials $\left(\chi^{2}=2.15, \mathrm{p}=0.54, \mathrm{I}^{2}=0 \%\right)$.

The analysis for total postoperative complications was based on 7 trials. EOF significantly reduced the risk of total postoperative complications compared with TOF (RR 0.70; 95\% CI 0.50-0.98; $\mathrm{p}=0.04$, from a fixed effects model), with no heterogeneity between trials $\left(\chi^{2}=2.07\right.$, $\left.\mathrm{p}=0.91, \mathrm{I}^{2}=0 \%\right)$ (fig. 3).

\section{Secondary Outcome Measures}

Vomiting was assessed in 4 studies. EOF did not increase the risk of vomiting compared with TOF (RR 1.08; $95 \%$ CI $0.77-1.53 ; \mathrm{p}=0.65)$, with little heterogeneity between trials $\left(\chi^{2}=4.62, \mathrm{p}=0.20, \mathrm{I}^{2}=35 \%\right)$. 


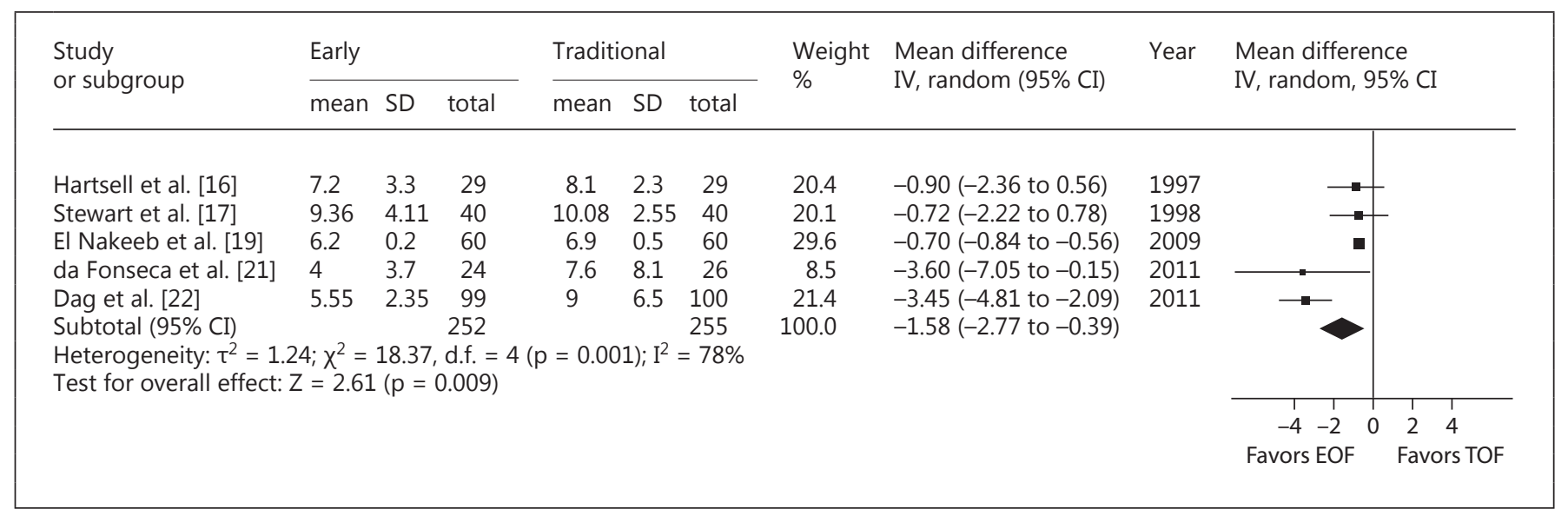

Fig. 2. EOF versus TOF for length of hospital stay (days).

NGT reinsertion was assessed in 5 studies. EOF did not increase the rate of NGT reinsertion compared with TOF (RR $1.31 ; 95 \%$ CI $0.78-2.21 ; \mathrm{p}=0.30$ ), with no heterogeneity between trials $\left(\chi^{2}=0.83, \mathrm{p}=0.93, \mathrm{I}^{2}=0 \%\right)$.

Mortality was assessed in 4 studies. EOF did not reduce or increase the risk of mortality compared with TOF (RR $0.61 ; 95 \%$ CI $0.15-2.50 ; \mathrm{p}=0.5$ ), with no heterogeneity between trials $\left(\chi^{2}=1.50, p=0.68, I^{2}=0 \%\right)$ (fig. 4).

Only 1 study assessed the financial impact of EOF and showed that hospital costs were similar between groups, with only USD 72 of savings in the EOF group [20].

None of the included studies assessed quality of life.

\section{Publication Bias}

We used the Egger weighted linear regression test to examine the asymmetry of funnel plots for all 8 metaanalysis outcomes [15]. There was no clear evidence of asymmetry in any of these plots (length of hospital stay, $\mathrm{p}=0.21$; anastomotic dehiscence, $\mathrm{p}=0.40$; pneumonia, $\mathrm{p}=0.28$; wound infection, $\mathrm{p}=0.32$; total postoperative complications, $\mathrm{p}=0.83$; vomiting, $\mathrm{p}=0.28$; NGT reinsertion, $\mathrm{p}=0.59$, and mortality, $\mathrm{p}=0.29$ ).

\section{Discussion}

The present meta-analysis showed that EOF was associated with a significant reduction in length of hospital stay and total postoperative complications compared with TOF in patients undergoing elective colorectal surgery. There were no significant differences in the risk of anastomotic dehiscence, pneumonia, wound infection, rate of NGT reinsertion, vomiting, or mortality.

EOF is one of several elements in fast-track surgery that can enhance recovery after colorectal resection [23, $24]$, and the evidence is mainly based on two meta-analyses $[3,4]$ (the latter study is essentially an updated metaanalysis of the earlier one). However, this evidence is inadequate. Firstly, both meta-analyses included many studies in which all or some patients had undergone other GI surgeries rather than colorectal surgery, e.g. upper GI surgery [25] and small bowel resection [26, 27]. Secondly, studies of oral feeding and tube feeding were combined in these meta-analyses. Fast-track colorectal surgery indicates early NGT removal, EOF, and early mobilization, rather than early tube feeding [23, 24]. Enteral routes of feeding like nasojejunal tubes or nasoduodenal tubes may lead to discomfort or inconvenience to patients. In addition, tube feeding may cause complications, such as tube malposition, aspiration pneumonia, sinusitis, epistaxis, tube occlusion [7-9], and even bowel necrosis [28], and it may influence postoperative outcomes. Furthermore, combining studies of oral feeding with studies of tube feeding may increase the heterogeneity between trials. Thirdly, the inclusion criteria of those two meta-analyses were not stringent. For example, both of the two meta-analyses included the study by Ortiz et al. [29], which compared not only EOF with TOF but also early versus late removal of NGT after colorectal surgery.

It has been reported that systematic use of NGT or fasting is still common in postoperative management of colorectal surgery $[30,31]$. This is the first meta-analysis to evaluate surgical outcomes following EOF compared 


\begin{tabular}{|c|c|c|c|c|c|c|c|c|c|c|}
\hline \multirow{2}{*}{$\begin{array}{l}\text { Study } \\
\text { or subgroup }\end{array}$} & \multicolumn{2}{|l|}{ Early } & \multicolumn{2}{|c|}{ Traditional } & \multirow{2}{*}{$\begin{array}{l}\text { Weight } \\
\%\end{array}$} & \multirow{2}{*}{$\begin{array}{l}\text { Risk ratio } \\
\mathrm{M}-\mathrm{H} \text {, fixed }(95 \% \mathrm{CI})\end{array}$} & \multirow[t]{2}{*}{ Year } & \multirow{2}{*}{\multicolumn{3}{|c|}{$\begin{array}{l}\text { Risk ratio } \\
M-H \text {, fixed, 95\% CI }\end{array}$}} \\
\hline & events & total & events & total & & & & & & \\
\hline \multicolumn{11}{|l|}{ Anastomotic dehiscence } \\
\hline Hartsell et al. [16] & 0 & 29 & 1 & 29 & 10.1 & $0.33(0.01-7.86)$ & 1997 & & & \\
\hline Stewart et al. [17] & 1 & 40 & 0 & 40 & 3.4 & $3.00(0.13-71.51)$ & 1998 & & & \\
\hline Lucha et al. [18] & 1 & 26 & 0 & 25 & 3.4 & $2.89(0.12-67.75)$ & 2005 & & & \\
\hline El Nakeeb et al. [19] & 1 & 60 & 2 & 60 & 13.5 & $0.50(0.05-5.37)$ & 2009 & & & \\
\hline da Fonseca et al. [21] & 0 & 24 & 4 & 26 & 29.2 & $0.12(0.01-2.12)$ & 2011 & & - & \\
\hline Dag et al. [22] & 2 & 99 & 6 & 100 & 40.3 & $0.34(0.07-1.63)$ & 2011 & & & \\
\hline Subtotal (95\% CI) & & 278 & & 280 & 100.0 & $0.47(0.19-1.15)$ & & & & \\
\hline Total events & 5 & & 13 & & & & & & & \\
\hline \multicolumn{11}{|c|}{ Heterogeneity: $x^{2}=3.67$, d.f. $=5(p=0.60) ; I^{2}=0 \%$} \\
\hline \multicolumn{11}{|c|}{ Test for overall effect: $Z=1.65(p=0.10)$} \\
\hline \multicolumn{11}{|l|}{ Pneumonia } \\
\hline Hartsell et al. [16] & 1 & 29 & 0 & 29 & 3.7 & $3.00(0.13-70.74)$ & 1997 & & & \\
\hline Stewart et al. [17] & 1 & 40 & 1 & 40 & 7.4 & $1.00(0.06-15.44)$ & 1998 & & & \\
\hline Lucha et al. [18] & 0 & 26 & 1 & 25 & 11.3 & $0.32(0.01-7.53)$ & 2005 & & & \\
\hline El Nakeeb et al. [19] & 2 & 60 & 7 & 60 & 51.8 & $0.29(0.06-1.32)$ & 2009 & & & \\
\hline Dag et al. [22] & 3 & 100 & 3 & 100 & 22.2 & $1.00(0.21-4.84)$ & 2011 & & & \\
\hline da Fonseca et al. [21] & 1 & 24 & 0 & 60 & 3.6 & $3.24(0.14-75.91)$ & 2011 & & & \\
\hline Subtotal $(95 \% \mathrm{CI})$ & & 279 & & 280 & 100.0 & $0.71(0.31-1.59)$ & & & & \\
\hline Total events & 8 & & 12 & & & & & & & \\
\hline \multicolumn{11}{|c|}{ Heterogeneity: $\chi^{2}=3.53$, d.f. $=5(p=0.62) ; I^{2}=0 \%$} \\
\hline \multicolumn{11}{|c|}{ Test for overall effect: $Z=0.84(p=0.40)$} \\
\hline \multicolumn{11}{|l|}{ Wound infection } \\
\hline Stewart et al. [17] & 0 & 40 & 4 & 40 & 24.5 & $0.11(0.01-2.00)$ & 1998 & & - & \\
\hline El Nakeeb et al. [19] & 5 & 60 & 5 & 60 & 27.2 & $1.00(0.31-3.28)$ & 2009 & & & \\
\hline Dag et al. [22] & 5 & 99 & 7 & 100 & 37.9 & $0.72(0.24-2.20)$ & 2011 & & - & \\
\hline da Fonseca et al. [21] & 2 & 24 & 2 & 26 & 10.4 & $1.08(0.17-7.10)$ & 2011 & & & \\
\hline Subtotal $(95 \% \mathrm{CI})$ & & 223 & & 226 & 100.0 & $0.69(0.34-1.37)$ & & & & \\
\hline Total events & 12 & & 18 & & & & & & & \\
\hline \multicolumn{11}{|c|}{ Heterogeneity: $\chi^{2}=2.15$, d.f. $=3(p=0.54) ; I^{2}=0 \%$} \\
\hline \multicolumn{11}{|c|}{ Test for overall effect: $Z=1.07(p=0.29)$} \\
\hline \multicolumn{11}{|l|}{ Total complications } \\
\hline Hartsell et al. [16] & 1 & 29 & 1 & 29 & 1.5 & $1.00(0.07-15.24)$ & 1997 & & & \\
\hline Stewart et al. [17] & 10 & 40 & 12 & 40 & 18.3 & $0.83(0.41-1.70)$ & 1998 & & & \\
\hline Lucha et al. [18] & 1 & 26 & 1 & 25 & 1.6 & $0.96(0.06-14.55)$ & 2005 & & & \\
\hline El Nakeeb et al. [19] & 14 & 60 & 22 & 60 & 33.5 & $0.64(0.36-1.12)$ & 2009 & & $=$ & \\
\hline Lobato Dias Consoli et al. [20] & 4 & 15 & 5 & 14 & 7.9 & $0.75(0.25-2.23)$ & 2010 & & & \\
\hline Dag et al. [22] & 12 & 99 & 14 & 100 & 21.2 & $0.87(0.42-1.78)$ & 2011 & & -1 & \\
\hline da Fonseca et al. [21] & 4 & 24 & 11 & 26 & 16.1 & $0.39(0.14-1.07)$ & 2011 & & -1 & \\
\hline Subtotal $(95 \% \mathrm{CI})$ & & 293 & & 294 & 100.0 & $0.70(0.50-0.98)$ & & & $\Delta$ & \\
\hline \multirow{2}{*}{\multicolumn{11}{|c|}{$\begin{array}{l}\text { Total events } \\
\text { Heterogeneity: } \chi^{2}=2.07, \text { d.f. }=6(p=0.91) ; I^{2}=0 \% \\
\text { Test for overall effect: } Z=2.11(p=0.04)\end{array}$}} \\
\hline & & & & & & & & & & \\
\hline & & & & & & & & $0.001 \quad 0.1$ & 10 & 10 \\
\hline & & & & & & & & Favors EOF & & Favors TOF \\
\hline
\end{tabular}

Fig. 3. EOF versus TOF for postoperative complications.

with TOF in patients undergoing elective colorectal surgery. Our results eliminate concerns long held by many surgeons that EOF may increase the incidence of anastomotic dehiscence and vomiting, and they provide support for surgeons to commence EOF after colorectal surgery.
The present study followed the recommendations of the PRISMA statement and was strengthened by the stringent inclusion criteria, rigorous search strategy, and avoidance of language limitation. In addition, this metaanalysis was based on 7 randomized controlled trials, and 


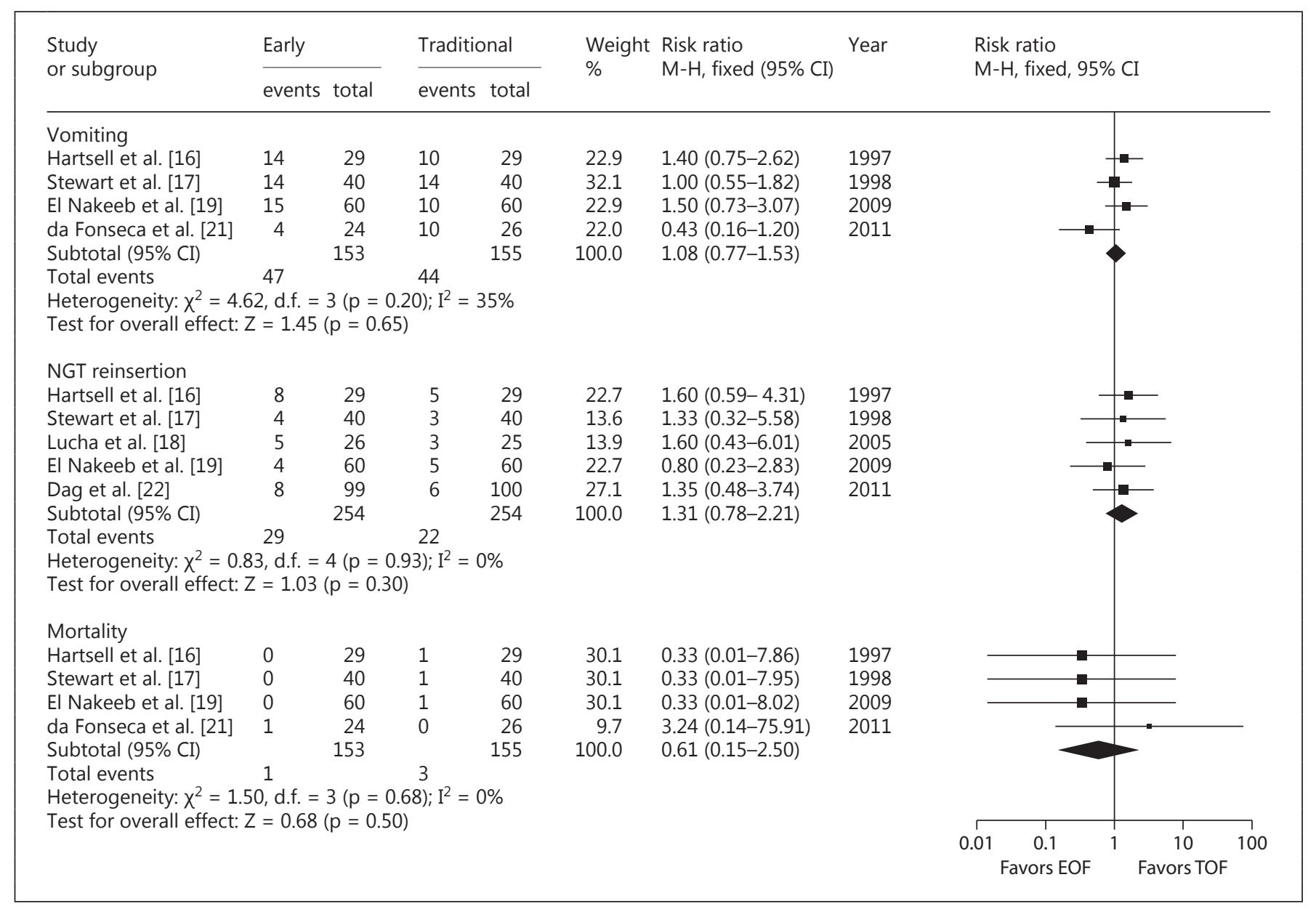

Fig. 4. EOF versus TOF for vomiting, NGT reinsertion, and mortality.

5 of these trials (449 patients) were published after 2005. In view of the surgical and anesthetic practices that have changed over recent years, the results of the present study will be more representative than the previous meta-analyses for current colorectal surgery.

There were several limitations to the present metaanalysis. First, 3 of 7 included studies were unclear in randomization sequence generations and, hence, selection bias or confounding might be present. In addition, none of the included studies used blinding for the observers or patients. For feeding protocols, however, they could not lend themselves to double (observers and patients) or single (patients only) blinding, as both would have detected the introduction of food. Second, high statistical heterogeneity was identified in the length of hospital stay. Differences in discharge criteria between the included trials may explain this heterogeneity. Third, the included stud- ies did not adequately evaluate hospital costs and quality of life after surgery, which are important outcomes for patients undergoing elective colorectal surgery. Finally, because only studies of EOF were included in this metaanalysis, our findings may not necessarily be generalized to patients with early postoperative tube feeding.

\section{Conclusions}

EOF after elective colorectal resection was beneficial and safe in enhancing recovery. EOF was associated with a lower incidence of postoperative complications and a reduction in length of hospital stay. Additional randomized controlled trials of EOF with long-term follow-up are necessary to assess hospital costs and quality of life in patients undergoing elective colorectal surgery. 


\section{Acknowledgement}

This work was partially supported by the Medical Supporting

Discipline Construction Program of Zhejiang Province (11-ZC24).

\section{Disclosure Statement}

All authors have no conflicts of interest to declare.

\section{References}

1 Bisgaard T, Kehlet H: Early oral feeding after elective abdominal surgery - what are the issues? Nutrition 2002;18:944-948.

2 Nelson R, Edwards S, Tse B: Prophylactic nasogastric decompression after abdominal surgery. Cochrane Database Syst Rev 2007: CD004929.

-3 Lewis SJ, Egger M, Sylvester PA, Thomas S: Early enteral feeding versus 'nil by mouth' after gastrointestinal surgery: systematic review and meta-analysis of controlled trials. BMJ 2001;323:773-776.

4 Andersen HK, Lewis SJ, Thomas S: Early enteral nutrition within $24 \mathrm{~h}$ of colorectal surgery versus later commencement of feeding for postoperative complications. Cochrane Database Syst Rev 2006, pp CD004080.

$\checkmark 5$ Lewis SJ, Andersen HK, Thomas S: Early enteral nutrition within $24 \mathrm{~h}$ of intestinal surgery versus later commencement of feeding: a systematic review and meta-analysis. J Gastrointest Surg 2009;13:569-575.

6 Osland E, Yunus RM, Khan S, Memon MA: Early versus traditional postoperative feeding in patients undergoing resectional gastrointestinal surgery: a meta-analysis. JPEN J Parenter Enteral Nutr 2011;35:473-487.

7 Gomes GF, Pisani JC, Macedo ED, Campos AC: The nasogastric feeding tube as a risk factor for aspiration and aspiration pneumonia. Curr Opin Clin Nutr Metab Care 2003;6:327333.

8 Ghahremani GG, Gould RJ: Nasoenteric feeding tubes: radiographic detection of complications. Dig Dis Sci 1986;31:574-585.

9 Levenson R, Turner WW Jr, Dyson A, Zike L, Reisch J: Do weighted nasoenteric feeding tubes facilitate duodenal intubations? JPEN J Parenter Enteral Nutr 1988;12:135-137.

$\checkmark 10$ Bourgault AM, Heyland DK, Drover JW, Keefe L, Newman P, Day AG: Prophylactic pancreatic enzymes to reduce feeding tube occlusions. Nutr Clin Pract 2003;18:398-401.

11 Moher D, Liberati A, Tetzlaff J, Altman DG: Preferred reporting items for systematic reviews and meta-analyses: the PRISMA statement. BMJ 2009;339:b2535.
12 Jadad AR, Moore RA, Carroll D, Jenkinson C, Reynolds DJ, Gavaghan DJ, McQuay HJ: Assessing the quality of reports of randomized clinical trials: is blinding necessary? Control Clin Trials 1996;17:1-12.

13 Huedo-Medina TB, Sanchez-Meca J, MarinMartinez F, Botella J: Assessing heterogeneity in meta-analysis: Q statistic or $\mathrm{I}^{2}$ index? Psychol Methods 2006;11:193-206.

14 Higgins JP, Thompson SG: Quantifying heterogeneity in a meta-analysis. Stat Med 2002; 21:1539-1558.

15 Egger M, Davey Smith G, Schneider M, Minder C: Bias in meta-analysis detected by a simple, graphical test. BMJ 1997;315:629-634.

16 Hartsell PA, Frazee RC, Harrison JB, Smith RW: Early postoperative feeding after elective colorectal surgery. Arch Surg 1997;132:518520, discussion 520-521.

17 Stewart BT, Woods RJ, Collopy BT, Fink RJ, Mackay JR, Keck JO: Early feeding after elective open colorectal resections: a prospective randomized trial. Aust N Z J Surg 1998;68: 125-128.

18 Lucha PA Jr, Butler R, Plichta J, Francis M: The economic impact of early enteral feeding in gastrointestinal surgery: a prospective survey of 51 consecutive patients. Am Surg 2005; 71:187-190.

19 El Nakeeb A, Fikry A, El Metwally T, Fouda E, Youssef M, Ghazy H, Badr S, Khafagy W, Farid M: Early oral feeding in patients undergoing elective colonic anastomosis. Int J Surg 2009;7:206-209.

20 Lobato Dias Consoli M, Maciel Fonseca L, Gomes da Silva R, Toulson Davisson Correia MI: Early postoperative oral feeding impacts positively in patients undergoing colonic resection: results of a pilot study. Nutr Hosp 2010;25:806-809.

21 da Fonseca LM, Profeta da Luz MM, LacerdaFilho A, Correia MI, Gomes da Silva R: A simplified rehabilitation program for patients undergoing elective colonic surgery - randomized controlled clinical trial. Int J Colorectal Dis 2011;26:609-616.
22 Dag A, Colak T, Turkmenoglu O, Gundogdu $\mathrm{R}$, Aydin S: A randomized controlled trial evaluating early versus traditional oral feeding after colorectal surgery. Clinics (Sao Paulo) 2011;66:2001-2005.

23 Kehlet H: Fast-track colorectal surgery. Lancet 2008;371:791-793.

24 Lassen K, Soop M, Nygren J, Cox PB, Hendry $\mathrm{PO}$, Spies C, von Meyenfeldt MF, Fearon KC, Revhaug A, Norderval S, Ljungqvist O, Lobo $\mathrm{DN}$, Dejong CH: Consensus review of optimal perioperative care in colorectal surgery: Enhanced Recovery After Surgery (ERAS) Group recommendations. Arch Surg 2009; 144:961-969.

25 Heslin MJ, Latkany L, Leung D, Brooks AD, Hochwald SN, Pisters PW, Shike M, Brennan MF: A prospective, randomized trial of early enteral feeding after resection of upper gastrointestinal malignancy. Ann Surg 1997;226: 567-577, discussion 577-580.

26 Binderow SR, Cohen SM, Wexner SD, Nogueras JJ: Must early postoperative oral intake be limited to laparoscopy? Dis Colon Rectum 1994;37:584-589.

27 Reissman P, Teoh TA, Cohen SM, Weiss EG, Nogueras JJ, Wexner SD: Is early oral feeding safe after elective colorectal surgery? A prospective randomized trial. Ann Surg 1995; 222:73-77.

28 Schloerb PR, Wood JG, Casillan AJ, Tawfik O, Udobi K: Bowel necrosis caused by water in jejunal feeding. JPEN J Parenter Enteral Nutr 2004;28:27-29.

29 Ortiz H, Armendariz P, Yarnoz C: Is early postoperative feeding feasible in elective colon and rectal surgery? Int J Colorectal Dis 1996;11:119-121.

-30 Kehlet H, Buchler MW, Beart RW Jr, Billingham RP, Williamson R: Care after colonic operation - is it evidence-based? Results from a multinational survey in Europe and the United States. J Am Coll Surg 2006;202:45-54.

- 31 Villalba Ferrer F, Bruna Esteban M, Garcia Coret MJ, Garcia Romero J, Roig Vila JV: Evidence of early oral feeding in colorectal surgery. Rev Esp Enferm Dig 2007;99:709-713. 